\title{
Une anthologie du Mugam d'Azerbaïdjan
}

\section{Pierre-Yves Haab}

\section{(2) OpenEdition \\ Journals}

Édition électronique

URL : http://journals.openedition.org/ethnomusicologie/1251

ISSN : 2235-7688

Éditeur

ADEM - Ateliers d'ethnomusicologie

Édition imprimée

Date de publication : 31 décembre 1995

Pagination : 261-264

ISBN : 2-8257-0537-3

ISSN : $1662-372 X$

Référence électronique

Pierre-Yves Haab, « Une anthologie du Mugam d'Azerbaïdjan », Cahiers d'ethnomusicologie [En ligne], 8 | 1995, mis en ligne le 04 janvier 2012, consulté le 30 avril 2019. URL : http:// journals.openedition.org/ethnomusicologie/1251

Ce document a été généré automatiquement le 30 avril 2019.

Tous droits réservés 


\title{
Une anthologie du Mugam d'Azerbaïdjan
}

\author{
Pierre-Yves Haab
}

\section{RÉFÉRENCE}

Alem Kassimov 1. Concerts des 10 et 12 mai 1989 à la MCM. 6 pages français/anglais. 2 transcriptions musicales. Durée : 72'12". 1 CD INEDIT W 260012, 1989.

Alem Kassimov 2. Concert du 11 mai 1989 à la MCM. 6 pages français/ anglais. 2 transcriptions musicales. Durée : 61'58". 1 CD INEDIT W 260015, 1990.

Hâji Bâbâ Huseynov. Concerts des 9, 10, 12 et 13 mars 1991 à la MCM. 14 pages français/ anglais. 3 transcriptions musicales. Durée : 57’47”. 1 CD INEDIT W 260026, 1991.

Trio Jabbâr Garyaghdu Oghlu. Concerts des 30 et 31 mai 1991 à la MCM. 14 pages français/ anglais. 2 transcriptions musicales. Durée : 68'05". 1 CD INEDIT W 260037, 1991.

Sakine Ismailova.Enregistré à Bakou en avril 1992. 16 pages français/ anglais. 2 transcriptions musicales. Durée : 71'48”. 1 CD INEDIT W 260049, 1993.

Aqakhân Abdullaev, Trio Zulfi Adigözelov. Enregistré à Bakou les 9 et 10 avril 1992. 16 pages français/anglais. 3 transcriptions musicales. Durée : 69'51". 1 CD INEDIT W 260052, 1994.

1 Inconnue des scènes occidentales il y a encore dix ans, la musique d'Azerbaïdjan a opéré depuis lors une percée significative auprès du public. Dès 1989, grâce à la Maison des Cultures du Monde, le groupe d'Alem Kassimov a rempli le rôle déterminant d'ambassadeur - un titre pleinement mérité en l'occurence - de cette tradition pluriséculaire située à la croisée de plusieurs cultures, mais dont le modèle persan reste cependant déterminant sur le plan strictement musical. Ceci n'était que le début d'une présentation qui allait bientôt être suivie d'autres concerts et déboucher sur l'édition de l'Anthologie du Mugam ${ }^{1}$ d'Azerbaïdjan : un fleuron de plus pour INEDIT - la collection de disques de la MCM - qui compte pour son dixième anniversaire une liste de titres prestigieux (dont deux autres anthologies, marocaine et tunisienne) récompensés de nombreux prix. 
2 Sur les six volumes publiés à ce jour, les quatre premiers sont le résultat des concerts donnés à la MCM entre 1989 et 1991, alors que les deux autres ont été enregistrés ${ }^{2}$ in situ, au Palais de la République à Bakou, de même que les trois volumes encore à paraître.

3 La notice d'accompagnement, de Pierre Bois, est comparable pour l'ensemble des volumes de cette anthologie: à part les deux premiers volumes présentant en une seule page l'histoire et la musique azéris, les musiciens, les règles mélodiques et les instruments, suivis de la transcription musicale des mugam, tous les autres comportent un texte identique, plus étoffé, sur l'histoire de l'Azerbaïdjan, l'art du Mugam, l'interprétation et les instruments ${ }^{3}$, auquel s'ajoute à chaque fois une notice particulière concernant les interprètes, dont on peut voir la photographie en page centrale. L'analyse des mugam, qui fait suite, est remarquable de fidélité : on y trouve non seulement le mode de base et les diverses modulations, mais également le découpage chronologique de l'exécution, avec mention de chaque passage (introduction, ghazal, tesnif, chant mesuré ou non, pièce instrumentale, changement de mode, etc.) et du rythme qui lui est propre. Ceci s'avère particulièrement efficace pour suivre avec précision chaque interprétation, a fortiori pour l'auditeur peu familiarisé avec la tradition azérie. A cela s'ajoute également la transcription ${ }^{4}$ des compositions vocales - chants de tradition populaire ou poèmes (ghazal ) faisant partie du patrimoine littéraire, dont certains, tels ceux de Nizâmi, remontent au $\mathrm{XII}^{\mathrm{e}}$ siècle. A lire ceux-ci, on regrette d'autant plus que la transcription ne soit pas toujours explicite. (Tous les textes ont-ils été traduits ? Comment sont-ils répartis? Se chantent-ils sur un seul passage ou se découpent-ils sur plusieurs d'entre eux ?) Cette critique mise à part, on a donc affaire, en quelques pages, à une présentation riche d'informations précieuses, qui saura satisfaire l'auditeur exigeant.

4 La tradition du Mugam d'Azerbaïdjan s'est définitivement fixée au XIX ${ }^{\mathrm{e}}$ siècle. C'est à cette époque que l'on a classé les onze modes principaux ou dastiakhi dans leurs limites actuelles et déterminé les modes secondaires. Le Mugam se présente comme une vaste composition vocale et instrumentale établie sur un mode de base, formée d'une suite de cadres mélodiques caratéristiques au rythme libre, où le chanteur peut donner cours avec une relative liberté à son improvisation, alternant avec des pièces mesurées vocales ( tesnif, ghazal) ou instrumentales (reng). Outre le mode de base, le cycle du Mugam passe en revue plusieurs modes secondaires qui lui sont apparentés et dont la succession est rigoureusement prédéterminée. Cette diversité, résultant du jeu des alternances rythmiques et de la subtile progression des altérations modales, s'offre en définitive comme « un flot musical proposant au détour de chaque méandre la contemplation d'un paysage inédit » - pour reprendre la belle expression de Pierre Bois.

5 L'interprétation du Mugam requiert toujours la même formation: un trio composé d'un chanteur s'accompagnant au daf (tambour sur cadre), d'un joueur de luth târ et d'un joueur de vièle kemânche, ces deux instruments étant identiques à ceux de la tradition persane. Cette musique savante ne semble, par ailleurs, pas connaître de problème de relève, si l'on en juge par la moyenne d'âge des exécutants figurant dans cette anthologie.

6 Agé de 32 ans à l'époque de l'enregistrement, Alem Kassimov est reconnu actuellement comme l'un des plus éminents chanteurs de Mugam de la nouvelle génération, apprécié pour la qualité de sa voix remarquablement souple jusque dans les registres les plus variés, et son travail tout en finesse qui confère une « précision quasi instrumentale » au chant. Il est accompagné au târ par Ekhan Mansurov et par Malik Mansurov au kemânche, tous deux fils de Bahram Mansurov, représentant d'une fameuse lignée de musiciens et mécènes de Bakou, dont Kassimov a été l'élève. 
7 Le volume 1 comporte les mugam Chargah et Bayati Shiraz, le volume 2 le mugam Rast et un tesnif (chant populaire) dans le mugam Dashti. Ce qui frappe dans ces deux enregistrements est l'impression qui ressort de parfaite cohésion entre les instrumentistes et le chanteur: un enrobement homogène de la voix par le târ et le kemânche, sans aucune " coupure ", qui témoigne de la profonde modestie des musiciens. Eu égard à la qualité musicale de ces prestations, on déplorera l'absence de toute transcription des textes chantés.

8 Avec le troisième volume nous est offert l'ultime document du dernier représentant de la vieille génération. Hâji Bâbâ Huseynov, né en 1919, nous l'a légué lors de son passage à Paris, avant de s'éteindre l'année suivante. Grand connaisseur de la poésie orientale, il a sauvé de l'oubli un nombre important de tesnif, composé également quelque 300 ghazal, et formé les jeunes espoirs de la génération actuelle. Dans ce mugam Humâyun, suivi de deux improvisations au târ par Aqasalim Abdullaev sur les modes Zâbol et Khârej Segâh enchaînant avec le mugam du même nom, on reconnaît un art parfaitement maîtrisé où la voix aux inflexions viriles et dénuée d'effets superflus veut avant tout rendre témoignage de la valeur poétique du texte, avec une sensibilité toute intériorisée.

9 Le Trio Jabbâr Garyaghdu Oghlu, dont le nom rend hommage à l'un des plus grand maitres du début du siècle, est constitué de Zayid Gouliev (chant), Mohled Mouslimov ( târ) et Fakhreddin Dadachev (kemânche). Dans ce volume, les mugam Shur et Mâhur Hindi sont développés avec grande délicatesse - comme on cisèle un joyau de prix - par une voix aux effets spectaculaires faisant preuve d'une intense expressivité, partagée par les instruments, qui s'élève au-dessus du strict académisme. Tout au long de leur interprétation, ces trois musiciens nous donnent l'agréable impression de partager un plaisir évident et démonstratif, duquel émane une joie généreuse et communicative.

10 Si le Mugam est principalement interprété par des hommes, le cinquième volume de cette collection nous rappelle toutefois que des femmes se distinguent également dans ce répertoire. Tout comme Zayid Gouliev, Sakine Ismaillova appartient à la génération d'Alem Kassimov. Membre de l'Opéra de Bakou, elle tient aux côtés de celui-ci tous les premiers rôles féminins des grands opéras-mugam. Considérée comme l'étoile montante du Mugam, elle doit son renom à une voix étonnamment souple, riche en timbres variés, capable des ornements les plus divers avec une égale légèreté. Accompagnée par Elkhan Muzafarov au târ et Arif Asadulaiev au kemânche, qui nous gratifient de deux splendides improvisations - le premier dans le mode Humayun, le second dans les modes Nava Nishapur et Ovshari -, elle interprète ici les mugam Mirza Husayn Segâh et Shahnaz, ainsi que trois zarbi mugam ou compositions vocales appartenant au genre « mugam mesuré ».

11 Le chant d'Aqakhân Abdullaev, dans le volume 6, se caractérise par un timbre à la fois riche et sobre, et une force intérieure s'éloignant de tout effet spectaculaire. Cet ancien élève de Hâji Bâbâ Huseynov, dont on reconnaît la rigueur classique, est accompagné de Zamik Aliev au târ et d'Adalat Vezirov au kemânche pour l'interprétation des mugam Segâh Zâbol, Rahâb , Bayâti Qâjâr et Ovshâri: un art d'humilité qui témoigne d'une grande maîtrise de la tradition du Mugam.

12 La parution successive de ces six volumes a été amplement célébrée dans les publications françaises - Le Monde de la Musique, Diapason et Télérama - par de nombreuses distinctions honorifiques. Nous ne doutons pas les trois prochains disques à venir ${ }^{5}$ nous réservent également des surprises de qualité. 


\section{NOTES}

1. Mugam (avec majuscule) indique le genre musical auquel est consacrée cette anthologie, alors que mugam désigne un mode mélodique de la tradition azérie.

2. Les enregistrements ont été réalisés par Pierre Simonin ; pour ceux de Bakou, vient s'ajouter la collaboration de Pierre Bois.

3. On trouve en plus, dans le volume 6 , une rubrique consacrée aux poètes dont sont interprétés les ghazal.

4. La traduction française des textes chantés est due aux soins de Tajahmadi.

5. Jeynal Akperov, Gandab Gulieva et Mele Khanom Eyyubova. 